\title{
Highlights from Studies in Cardiovascular Disease Prevention Presented at the Digital 2020 European Society of Cardiology Congress: Prevention Is Alive and Well
}

\author{
Xiaoming Jia ${ }^{1} \cdot$ Mahmoud Al Rifai $^{1} \cdot$ Aliza Hussain $^{1}$ - Seth Martin ${ }^{2} \cdot$ Anandita Agarwala $^{3} \cdot$ Salim S. Virani ${ }^{1,4,5}$ (D)
}

Accepted: 16 September 2020 / Published online: 3 October 2020

(C) This is a U.S. Government work and not under copyright protection in the US; foreign copyright protection may apply 2020

\begin{abstract}
Purpose of Review The review highlights selected studies related to cardiovascular disease (CVD) prevention that were presented at the 2020 European Society of Cardiology (ESC) Congress-The Digital Experience.

Recent Findings The studies reviewed include clinical trials on novel RNA interference-based lipid-lowering therapies AKCEA-APOCIII- $\mathrm{L}_{\mathrm{Rx}}$ and vupanorsen (AKCEA-ANGPTL3- $\mathrm{L}_{\mathrm{Rx}}$ ); the EVAPORATE trial assessing the effects of icosapent ethyl on coronary plaque volume progression; the LoDoCo2 trial evaluating the efficacy of low-dose colchicine in cardiovascular disease risk reduction among patients with chronic coronary artery disease; as well as the EMPEROR-Reduced trial evaluating cardiovascular and renal outcomes with empagliflozin in patients with heart failure and reduced ejection fraction. In addition, we review the BPLTTC analysis on blood pressure treatment across blood pressure levels and CVD status and discuss findings from the BRACE CORONA study that examined continuing versus suspending angiotensin-converting enzyme inhibitor or angiotensin receptor blockers in patients on these antihypertensive medications who were hospitalized with COVID-19 infection.

Summary The studies presented at the 2020 digital ESC Congress highlight the continuing advancements in the field of CVD prevention.
\end{abstract}

Keywords Cardiovascular disease prevention · APOCIII · ANGPTL3 · Colchicine $\cdot$ Empagliflozin $\cdot$ COVID-19

Xiaoming Jia, Mahmoud Al Rifai and Aliza Hussain contributed equally to this work.

This article is part of the Topical Collection on Reviews and New Research Implications

Salim S. Virani

virani@bcm.edu

Xiaoming Jia

Xiaoming.Jia@bcm.edu

Mahmoud Al Rifai

Mahmoud.AlRifai@bcm.edu

Aliza Hussain

Aliza.Hussain@bcm.edu

Seth Martin

smart100@jhmi.edu

Anandita Agarwala

anandita@wustl.edu
1 Section of Cardiology, Baylor College of Medicine, Houston, TX, USA

2 Department of Medicine, Division of Cardiology, Ciccarone Center for the Prevention of Cardiovascular Disease, Johns Hopkins University School of Medicine, Baltimore, MD, USA

3 Baylor Scott and White Health Heart Hospital Baylor Plano, Plano, TX, USA

4 Health Policy, Quality \& Informatics Program, Health Services Research and Development Center for Innovations, Michael E. DeBakey Veterans Affairs Medical Center, 2002 Holcombe Boulevard, Houston, TX 77030, USA

5 Section of Cardiology, Michael E. DeBakey Veterans Affairs Medical Center, Houston, TX, USA 


\section{Introduction}

Novel advancements in the field of cardiovascular disease (CVD) prevention were recently showcased at the 2020 European Society of Cardiology Congress (ESC Congress 2020).

Due to the ongoing coronavirus disease 2019 (COVID-19) pandemic, the scientific session was held on a digital platform, allowing for the presentation and discussion of the latest science in cardiology despite the COVID-19 outbreak. Below, we summarize findings from seven major studies relevant to
CVD prevention that were presented at ESC Congress 2020 (Table 1).

The studies include clinical trials of novel lipid-lowering therapies AKCEA-APOCIII- $\mathrm{L}_{\mathrm{Rx}}$ and vupanorsen (AKCEAANGPTL3- $\mathrm{L}_{\mathrm{Rx}}$ ). We further highlight data from the Effect of Vascepa on Improving Coronary Atherosclerosis in People With High Triglycerides Taking Statin Therapy (EVAPORATE) study that evaluated the effect of icosapent ethyl on coronary plaque volume; findings from the Low Dose Colchicine 2 (LoDoCo2) trial assessing the efficacy of colchicine in cardiovascular disease risk reduction among patients

Table 1 Summary of the trials reviewed in this article

\begin{tabular}{|c|c|c|c|c|c|}
\hline Study & Intervention & Design & $\mathrm{N}$ & $\begin{array}{l}\text { Follow-up } \\
\text { duration }\end{array}$ & Principle findings \\
\hline $\begin{array}{l}\text { AROAPOC } 331001 \\
\quad(8)\end{array}$ & $\begin{array}{l}\text { SiRNA } \\
\quad \text { targeting } \\
\text { APOC3 }\end{array}$ & $\begin{array}{l}\text { Open-label, phase I clinical } \\
\text { trial in healthy human } \\
\text { volunteers }\end{array}$ & 12 & 16 weeks & $\begin{array}{l}\text { Mean max change: } \\
\text { 1. APOC } 3:-94 \% \\
\text { 2. TG: }-75 \% \\
\text { 3. LDL-C: }-25 \% \\
\text { 4. apoB: }-33 \% \\
\text { 5. HDL-C: }+75 \% \\
\text { No SAE. } 2 / 12(17 \%) \text { with ISR }\end{array}$ \\
\hline AROANG1001 [1] & $\begin{array}{l}\text { SiRNA } \\
\text { targeting } \\
\text { ANGPTL3 }\end{array}$ & $\begin{array}{l}\text { Open-label, phase I clinical } \\
\text { trial in healthy human } \\
\text { volunteers }\end{array}$ & 12 & 16 weeks & $\begin{array}{l}\text { Mean max change: } \\
\text { 1. ANGPTL3: }-96 \% \\
\text { 2. TG: }-72 \% \\
\text { 3. LDL-C: }-50 \% \\
\text { 4. apoB: }-49 \% \\
\text { 5. non-HDL-C: }-54 \% \\
\text { 6. HDL-C: }-47 \% \\
\text { No SAE. Headache and URI most common AE }\end{array}$ \\
\hline EVAPORATE [2] & $\begin{array}{l}\text { Icosapent } \\
\text { ethyl } 4 \mathrm{~g} \\
\text { daily }\end{array}$ & $\begin{array}{l}\text { Randomized, double-blind, } \\
\text { placebo-controlled trial }\end{array}$ & 80 & 18 months & $\begin{array}{l}\text { Change in LAP volume: }-0.3 \pm 1.5 \mathrm{~mm}^{3} \text { in IPE vs. } \\
0.9 \pm 1.7 \mathrm{~mm}^{3} \text { in placebo, } p=0.006\end{array}$ \\
\hline LoDoCo2 [3] & $\begin{array}{l}\text { Colchicine } \\
\quad 0.5 \mathrm{mg} \\
\text { daily }\end{array}$ & $\begin{array}{l}\text { Randomized, double-blind, } \\
\text { placebo-controlled trial }\end{array}$ & 5522 & 28.6 months & $\begin{array}{l}\text { Primary composite endpoint (CV death, spontaneous } \\
\text { MI, ischemic stroke, or ischemia-driven coronary } \\
\text { revascularization): } \\
\text { HR } 0.69,95 \% \text { CI } 0.57-0.83, p<0.001\end{array}$ \\
\hline BPLTTC [4] & $\begin{array}{l}\text { Blood pressure } \\
\text { lowering }\end{array}$ & $\begin{array}{l}\text { Meta-analysis of } \\
\text { individual-level data from } \\
48 \text { clinical trials of blood } \\
\text { pressure lowering }\end{array}$ & 348,854 & 4 years & $\begin{array}{l}10 \% \text { risk reduction in major } \mathrm{CV} \text { outcomes, } 13 \% \\
\text { lower risk for stroke, } 7 \% \text { for ischemic heart } \\
\text { disease, } 14 \% \text { for } \mathrm{HF} \text {, and } 5 \% \text { for death per } \\
5 \mathrm{mmHg} \text { reduction in } \mathrm{SBP}\end{array}$ \\
\hline $\begin{array}{l}\text { EMPEROR-Reduced } \\
\text { [5] }\end{array}$ & $\begin{array}{l}\text { Empagliflozin } \\
10 \mathrm{mg} \text { daily }\end{array}$ & $\begin{array}{l}\text { Randomized, double-blind, } \\
\text { placebo-controlled trial }\end{array}$ & 3730 & 16 months & $\begin{array}{l}\text { Primary composite endpoint (CV death or HF } \\
\quad \text { hospitalization): } \\
\text { HR } 0.75,95 \% \text { CI } 0.65-0.86, p<0.001\end{array}$ \\
\hline $\begin{array}{l}\text { BRACE CORONA } \\
\text { [6] }\end{array}$ & $\begin{array}{l}\text { Continuing vs. } \\
\text { suspending } \\
\text { ACEI/ARB }\end{array}$ & Phase IV randomized trial & 659 & 30 days & $\begin{array}{l}\text { Primary outcome (days alive and out of hospital at } \\
30 \text { days): } \\
21.9 \pm 8.0 \text { mean days in continuing ACEI/ARB } \\
\text { group vs. } 22.9 \pm 7.1 \text { mean days in suspending } \\
\text { ACEI/ARB group (mean difference between } \\
\text { groups was }-1.1 \text { days, } 95 \% \text { CI }-2.33-0.17 \text { ). } \\
\text { Mean ratio } 0.95,95 \% \text { CI } 0.90-1.01, p=0.09\end{array}$ \\
\hline
\end{tabular}

Abbreviations: $A E$ serious adverse events, apoB apolipoprotein $\mathrm{B}$, apoC-III apolipoprotein $C$-III, $A N G P T L 3$ angiopoietin-like protein 3, $H D L-C$, highdensity lipoprotein cholesterol, $T G$ triglycerides, $n o n-H D L-C$ non-high-density lipoprotein cholesterol, $L D L-C$ low-density lipoprotein cholesterol, $S A E$ serious adverse events, $I S R$ injection site reactions, $U R I$ upper respiratory tract infection, $L A P$ low-attenuation plaque, IPE icosapent ethyl, $C V$ cardiovascular, $M I$ myocardial infarction, $H R$ hazard ration, $C I$ confidence interval, $H F$ heart failure, $S B P$ systolic blood pressure, $A C E I$ angiotensinconverting enzyme inhibitor, ARB angiotensin receptor blocker 
with chronic coronary artery disease; as well as the Empagliflozin Outcome Trial in Patients with Chronic Heart Failure with Reduced Ejection Fraction (EMPERORReduced) trial evaluating cardiovascular (CV) and renal outcomes with empagliflozin in heart failure. In addition, we review the Blood Pressure Lowering Treatment Trialists Collaboration (BPLTTC) analysis on blood pressure treatment across blood pressure levels and CVD status. Finally, we outline findings from the Angiotensin Receptor Blockers and Angiotensin-converting Enzyme Inhibitors and Adverse Outcomes in Patients With COVID19 (BRACE CORONA) trial that examined continuing versus suspending angiotensin-converting enzyme inhibitors (ACEI) or angiotensin receptor blockers (ARB) in patients hospitalized with COVID-19 infection. In this review, we will to provide concise summaries of the major findings from these studies, put these findings in the context of what is known on the topic, and discuss their clinical implications [7-9].

\section{RNA Interference Therapies Targeting Novel Triglyceride Pathways: ARO-APOC3 and ARO-ANG3}

Lipoprotein lipase (LPL) is a key enzyme involved in the clearance of triglycerides (TG) and triglyceride-rich lipoproteins (TGRL) from the blood circulation. Apolipoprotein C-III (apoC-III) and angiopoietin-like protein 3 (ANGPTL3) are hepatic secretory proteins that inhibit the activity of LPL and consequently increase TG levels. ApoC-III also decreases the uptake and clearance of TGRL via LPL-independent mechanisms, with an associated increase in very low-density lipoprotein (VLDL) and decrease in high-density lipoprotein (HDL). Loss of function mutations in ANGPTL3 results in reduction in low-density lipoprotein (LDL), VLDL, HDL, and TG. Mendelian randomization studies have shown that polymorphisms of the genes encoding these proteins are causally associated with atherosclerotic cardiovascular disease (ASCVD) [10, 11]. Considerable interest has been shown in the development of novel agents for these potential therapeutic targets [12].

RNA interference (RNAi) or post-transcriptional gene silencing is a biological process whereby small RNA molecules inhibit gene expression or translation by binding to and neutralizing messenger RNA (mRNA) molecules. Anti-sense oligonucleotide (ASO) and small interfering RNA (siRNA) are two types of RNAi technology currently being employed to target production of apoC-III and ANGPTL3 and thereby, lower levels of TG. SiRNA is a double-stranded RNA, which upon entry into the cytoplasm, hybridizes and degrades mRNA [13].
Study Overview: RNAi Targeting apoC-III with AROAPOC 3 in Healthy Volunteers

ARO-APOC3 is a siRNA that inhibits the production of apoC-III. This in turn increases the hydrolysis of TGRL via LPL, resulting in increased uptake and clearance of TGRL with an overall reduction in TG and VLDL and increase in HDL-C. AROAPOC331001 is the first in human study to evaluate the efficacy, safety, and tolerability of AROAPOC3 in healthy volunteers [14].

In an open-label clinical trial, 12 healthy volunteers were administered $10 \mathrm{mg}(n=4), 25 \mathrm{mg}(n=4)$, and $50 \mathrm{mg}(n=4)$ of ARO-APOC 3 by subcutaneous injection on day 1 and day 29 (week 4). Over a 16-week follow-up period, repeat doses of ARO-APOC3 demonstrated substantial and durable mean reductions in apoC-III levels by $73 \%$ with $10 \mathrm{mg}, 90 \%$ with $25 \mathrm{mg}$, and $94 \%$ with $50 \mathrm{mg}$ dosage and fasting TG levels by $58 \%(10 \mathrm{mg}), 70 \%(25 \mathrm{mg})$, and $75 \%$ (50 mg), respectively. Fasting low-density lipoprotein cholesterol (LDL-C) levels were reduced by $20-25 \%$, apolipoprotein B (apoB) levels by 28-33\%, while high-density lipoprotein cholesterol (HDL-C) levels increased by $30-74 \%$. Moreover, there was a reduction in both fasting TG levels as well as post-prandial lipemia.

ARO-APOC3 had a favorable safety and tolerability profile. All adverse events (AE) were mild, with the most common being diarrhea (33\%). Local injection site reactions occurred in 2/12 (17\%) of the participants with most injection site AE resolving within $48 \mathrm{~h}$. There were no reported serious AEs and no clinically significant changes in platelets, bilirubin, creatinine, or liver enzymes.

\section{Study Overview: RNAi Targeting ANGPTL3 with ARO- ANG3 in Healthy Volunteers}

ARO-ANG3 is a siRNA directed to hepatocytes where it specifically degrades the mRNA of ANGPTL3, resulting in gene silencing. AROANG1001 is a first in human study carried out in healthy volunteers to study the pharmacokinetics, safety, and tolerability of ARO-ANG3 [1].

In an open-label trial, 12 healthy volunteers were administered $100 \mathrm{mg}(n=4), 200 \mathrm{mg}(n=4)$, and $300 \mathrm{mg}(n=4)$ of multiple subcutaneous doses of ARO-ANG3 on day 1 and day 29 (week 4). Substantial and durable mean reductions were demonstrated in ANGPTL3 levels by $81 \%$ (100 mg), $86 \%$ (200 mg), and $96 \%$ (300 mg) and fasting TG by $62 \%$ (100 mg), 72\% (200 mg), and 67\% (300 mg), respectively. It also demonstrated a mean maximum reduction in LDL-C by $50 \%$, apoB by $42 \%$, non-HDL-C by $54 \%$, and HDL-C by $47 \%$ and showed improvement in post-prandial lipemia.

ARO-ANG3 was well-tolerated without any serious adverse events with no significant discontinuation of the study medication. The most common adverse events were headache $(5 / 12,42 \%)$, upper respiratory tract infection $(4 / 12,33 \%)$, and 
vascular access bruising/swelling (4/12, 33\%). Injection site reactions occurred in 2/12 participants (17\%). There were no clinically significant adverse effects on the levels of platelets, total bilirubin, creatinine, or transaminases.

\section{Clinical Implications}

There is increasing recognition of the causal role of TGRL in the pathogenesis of ASCVD and TG as a potential marker for increased ASCVD risk. As a result, there has been a growing focus on developing TG-lowering strategies with potential for $\mathrm{CV}$ benefit. Pharmacological inhibition of apoC-III with ARO-APOC3 produced favorable lipid changes, with substantial and durable reduction in TG, apoC-III, and LDL-C; increase in HDL-C; and improvement in post-prandial lipemia. Similarly, a repeated dose of ARO-ANGPTL3 also demonstrated significant dose-dependent reduction in fasting ANGPTL3, TG LDL-C, apoB, and non-HDL-C. One difference was a decrease, as opposed to increase, in HDL-C. Furthermore, both ARO-APOC3 and ARO-ANGPTL3 demonstrated acceptable safety and tolerability profile without any serious adverse events. In individuals treated with optimal statin and other evidence-based lipid-lowering therapies, considerable residual ASCVD risk persists [15••]. ApoC-III and ANGPTL3 inhibition have the potential to effectively treat combined hyperlipidemia and decrease residual risk in patients with ASCVD beyond current guideline-recommended standard of care. In addition, significant LDL-C reduction with ANGPTL3 inhibition appears to be independent of the LDL receptor pathway. Therefore, ANGPTL3 inhibitors may serve as an important adjunct in the treatment of homozygous FH patients with mutations in the LDL receptor pathway [16]. While the results from both of these studies are promising, they should be viewed with caution as this technology is novel, and we await more data from future phase II and III clinical trials to ascertain their effects on CV outcomes and establish long-term efficacy, safety, and tolerability before this technology can be integrated into routine clinical practice.

\section{Effect of Icosapent Ethyl on Progression of Coronary Atherosclerosis in Patients with Elevated Triglycerides on Statin Therapy: Final Results of the EVAPORATE Trial}

Icosapent ethyl (IPE) is a highly purified form of eicosapentaenoic acid. In the Reduction of Cardiovascular Events with Icosapent Ethyl-Intervention Trial (REDUCEIT), individuals with either established ASCVD or with diabetes and additional CV risk factors on stable statin therapy (with or without ezetimibe) were randomized to IPE $4 \mathrm{~g}$ daily versus placebo. Over a median follow-up period of 4.9 years, participants in the IPE group showed reduced adverse CV outcomes when compared with placebo ( $4.3 \%$ vs. $5.2 \%$; hazard ratio (HR) $0.80,95 \%$ confidence interval $(\mathrm{CI}) 0.66$ to 0.98 , $p=0.03)[17 \bullet]$. The EVAPORATE trial examined the effect of IPE on coronary plaque progression among patients with persistently elevated triglycerides on statin therapy [2]. The present study sought to clarify the mechanism of benefit of IPE which was shown to reduce CV events in the REDUCEIT trial.

\section{Study Overview}

EVAPORATE was a randomized, double-blind, placebocontrolled trial of 80 patients aged 30-85 years with known coronary atherosclerosis (narrowing of $\geq 20 \%$ in 1 coronary artery by either invasive angiography or coronary computed tomography angiography CCTA), elevated fasting triglyceride levels $(135-499 \mathrm{mg} / \mathrm{dL})$, and LDL-C levels between $\geq 40$ and $\leq 115 \mathrm{mg} / \mathrm{dL}$. Patients were required to be on statin therapy, with or without ezetimibe, and demonstrated adherence to therapeutic lifestyle changes including diet and exercise. The IPE dose was $2 \mathrm{~g}$ twice daily with food. The primary endpoint was the change in low-attenuation plaque (LAP) volume measured using multidetector computed tomography (MDCT) angiography at 18 months. Percent change in plaque characteristics was compared using multiple linear regression adjusted for baseline plaque, age, sex, diabetes status, hypertension, and baseline triglyceride levels using an intention-totreat analysis.

The mean age of study participants was $57 \pm 6$ years, $54 \%$ men, $82 \%$ white, $69 \%$ with diabetes mellitus, $77 \%$ with hypertension, and $43 \%$ with prior cigarette smoking history. Mean triglyceride level was $259 \pm 78 \mathrm{mg} / \mathrm{dL}$, with no significant differences between the 2 groups. Change in LAP volume was significantly lower among those treated with IPE ($\left.0.3 \pm 1.5 \mathrm{~mm}^{3}\right)$ versus mineral oil placebo $\left(0.9 \pm 1.7 \mathrm{~mm}^{3}\right)$ $(p=0.006)$. Other plaque characteristics were also improved in the IPE group including change in total plaque volume ($9 \%$ vs. $+11 \%, p=0.002)$, change in total non-calcified plaque volume $(-19 \%$ vs. $+9 \%, p=0.0005)$, change in fibrofatty plaque $(-34 \%$ vs. $+32 \%, p=0.0002)$, change in fibrous plaque $(-20 \%$ vs. $1 \%, p=0.003)$, and change in calcified plaque $(-1 \%$ vs. $+15 \%, p=0.053)$. Notably, there were no significant differences in total cholesterol, LDL-C, highdensity lipoprotein cholesterol, and triglyceride levels from baseline to follow-up between the two groups.

\section{Clinical Implications}

The EVAPORATE trial demonstrates that IPE reduces CV outcomes likely, at least in part, by slowing the progression of high-risk atherosclerotic plaques. IPE decreased the progression of LAP, which reflects plaques that are more prone to rupture. Prior studies have demonstrated that statins and 
proprotein convertase subtilisin/kexin type 9 (PCSK9) inhibitors also reduce coronary plaque progression $[18,19]$. The benefits of lipid-lowering drugs on CV outcomes are likely in part mediated by their effects on coronary plaques with a decrease in the number of plaques that are likely to rupture and a shift to and resultant increase in those that are more stable (calcified).

The clinical use of CCTA at present is mainly for risk prediction in the primary prevention of ASCVD. Coronary artery calcium (CAC) is assigned a IIA recommendation for additional risk stratification among intermediate-risk patients after evaluating risk-enhancing factors [20••]. At presents, only LDL-C is used to assess the response and adherence to lipid-lowering therapy. Future studies are warranted to investigate the potential role of CCTA or other measurements of atherosclerotic plaque progression among high-risk patients in order to document response to treatment. These benefits must be weighed against the inherent risks of contrast, exposure to radiation, and cost. The anatomic approach of evaluating subclinical atherosclerosis using CCTA and CAC is quite promising for both primary and secondary prevention of ASCVD.

\section{Colchicine in Patients with Chronic Coronary Disease: the LoDoCo2 Trial}

Residual ASCVD risk exists even among patients who are optimally treated with guideline-directed medical therapy, including statins, antiplatelet agents, and treatment of other risk factors. A growing body of evidence suggests that increased inflammation is causally associated with risk for ASCVD [21]. The Canakinumab Anti-inflammatory Thrombosis Outcome Study (CANTOS) demonstrated that treatment of secondary prevention patients with elevated inflammation as measured by elevated high-sensitivity C-reactive protein (hsCRP) with canakinumab resulted in significantly lower rates of recurrent cardiovascular events compared with placebo [22•]. The Low Dose Colchicine (LoDoCo) trial was an open-label study of 531 participants that showed a reduced risk for acute CVD events in individuals with chronic coronary artery disease treated with colchicine (HR $0.33,95 \%$ CI $0.18-0.59, p<0.001)$ [23]. The Colchicine Cardiovascular Outcomes Trial (COLCOT) demonstrated the effect of colchicine for cardiovascular risk reduction among patients with recent myocardial infarction (MI) within the past 30 days [24•]. In COLCOT, cardiovascular event rates were 5.5\% among patients in the colchicine group, compared with $7.1 \%$ in the placebo group (hazard ratio, $0.77 ; 95 \%$ CI 0.61 to 0.96 ; $p=0.02$ ). The LoDoCo 2 trial built upon the results of the LoDoCo and COLCOT studies and examined the effect of colchicine on cardiovascular events among a large sample of patients with chronic/stable coronary artery disease (CAD) [3].

\section{Study Overview}

LoDoCo2 was a randomized, double-blind, placebocontrolled trial of 5522 patients aged 35-82 years with stable coronary artery disease and no acute cardiovascular events in the 6 months prior to randomization. Patients were excluded if they had severe valvular heart disease, severe heart failure, moderate-to-severe renal impairment, or known side effects from colchicine. The presence of $\mathrm{CAD}$ was documented using prior invasive coronary angiography or CCTA or a CAC score of at least 400 Agatston units. All patients underwent a 1month run-in phase where they received $0.5 \mathrm{mg}$ of colchicine daily and then were subsequently randomized in a 1:1 fashion to receive $0.5 \mathrm{mg}$ of colchicine once daily or matching placebo. The primary endpoint was a composite of $\mathrm{CV}$ death, spontaneous (nonprocedural) MI, ischemic stroke, or ischemiadriven coronary revascularization. Cox regression models were used to study the association between colchicine versus placebo and the primary outcomes using an intention-to-treat analysis.

The mean age of the study participants was $66 \pm 8.6$ years, $15 \%$ were women, $12 \%$ were current smokers, and $18 \%$ had diabetes mellitus. The majority of patients treated with colchicine were on guideline-directed medical treatment of antiplatelets (67\%), lipid-lowering drugs (97\%), anticoagulants $(12 \%)$, and antihypertensive agents (72\% reninangiotensin inhibitors, $61 \%$ beta-blocker, $23 \%$ calcium channel blocker). The majority of patients $(84 \%)$ had a history of acute coronary syndrome with $68 \%$ having had an event at least 24 months before randomization. The distribution of baseline characteristics was equal between the 2 groups.

Over a median follow-up duration of 29 months, a total of 451 composite events occurred, with $6.8 \%$ in the colchicine group and $9.6 \%$ in the placebo group. The absolute event rates per 100 person-years were 2.5 and 3.6, respectively (HR 0.69 , $95 \%$ CI $0.57-0.83, p<0.001)$. This was mainly driven by a reduction in MI and ischemia-driven coronary revascularization. Non-CV deaths occurred more frequently among the colchicine group, but this was not significant. There were similar rates of cancer diagnosis, hospitalization for infection, hospitalization for pneumonia, and hospitalization for a gastrointestinal reason in either group. Neutropenia and myotoxic effects were overall low in both groups and not significantly different. Gout occurred significantly less frequently among those treated with colchicine (cumulative incidence ratio, $0.40 ; 95 \% \mathrm{CI}, 0.28$ to 0.58 ). The effect of colchicine as compared with placebo was consistent in prespecified subgroup analyses.

\section{Clinical Implications}

Results from the LoDoCo2 study further solidifies inflammation as an important risk for ASCVD and provides evidence 
supporting use of anti-inflammatory agents to target this risk. Colchicine is a commonly prescribed, well-established medication, which may facilitate the repurposing of this agent for use in CVD prevention. Importantly, the safety of antiinflammatory agents must be well established particularly with regard to non-cardiovascular outcomes. However, unlike canakinumab, which is known to target a specific inflammatory pathway, the mechanism of action for colchicine in the reduction of ASCVD remains unclear. Moreover, inflammatory markers such as hs-CRP were not measured routinely in LoDoCo2, and therefore, future studies are warranted to identify potential biomarkers that can used to better stratify patients who most benefit from colchicine.

\section{Blood Pressure Lowering Treatment Trialists' Collaboration: BPLTTC Trial}

\section{Study Overview}

The BPLTTC trial sought to examine the effect of blood pressure lowering for the prevention of ASCVD outcomes among patients with normal blood pressure (BP). Prior studies have yielded inconsistent results leading to contradictory treatment recommendations.

BPLTTC researchers conducted a meta-analysis of individual-level data from 48 clinical trials worldwide [4]. The sample size included 348,854 participants, and the average duration of follow-up was 4 years. Participants were divided into 2 groups based on a prior diagnosis of ASCVD. Each group was further divided into 7 subgroups based on categories of systolic blood pressure (SBP) at study entry (< $120,120-129,130-139,140-149,150-159,160-169$, and $\geq$ $170 \mathrm{mmHg}$ ).

Each $5 \mathrm{mmHg}$ reduction in SBP was associated with a $10 \%$ relative risk reduction of major cardiovascular outcomes including a $13 \%$ lower risk for stroke, $7 \%$ for ischemic heart disease, $14 \%$ for heart failure, and $5 \%$ for death. Importantly, there was no effect modification with respect to relative risk reduction (per $5 \mathrm{mmHg}$ ) by presence of ASCVD or SBP category at study entry. However, it should be noted that in patients with higher baseline SBP, achieving a greater magnitude of change in SBP will likely be associated with a greater absolute risk reduction in cardiovascular events.

\section{Clinical Implications}

Current ACC/AHA hypertension guidelines from 2017 recommend initiation of BP-lowering therapy among patients with $\mathrm{BP} \geq 140 / 90 \mathrm{mmHg}$ or with $130-189 / 80-89 \mathrm{mmHg}$ if they have clinical ASCVD or $\geq 10 \%$ estimated risk of ASCVD in those without prior ASCVD with a BP treatment goal of $<130 / 80 \mathrm{mmHg}[25 \bullet \bullet$ ]. Based on results of the
BPLTTC study, the decision to prescribe BP-lowering therapy may not be based on a prior diagnosis of ASCVD or current $\mathrm{BP}$ levels as there is no evidence that the effects of BPlowering drugs differ by baseline SBP values even as low as $<120 \mathrm{mmHg}$ among both primary and secondary prevention populations.

The BPLTTC study challenges the current paradigm of hypertension treatment. The current approach to BP lowering is not only based on BP criteria and clinical characteristics of patients including the presence of ASCVD but also incorporates risks of aggressive BP lowering including effects on kidney disease progression and serious safety outcomes such as falls. Furthermore, the effect of BP lowering on diastolic blood pressure (DBP) and its subsequent effect of cardiovascular outcomes needs to be further studied to document the safety of aggressive DBP lowering. Until more information is available, clinicians should continue to adhere to current guidance on BP lowering using a shared decision-making approach incorporating patient values and preferences. BP should be measured at every visit to assess response and adherence to treatment. Based on the present study, it may be reasonable to continue the present dose of BP-lowering drugs among patients who achieve SBP $<120 \mathrm{mmHg}$ and are tolerating therapy well without side effects or adverse outcomes.

\section{EMPEROR-Reduced: Empagliflozin in Heart Failure with a Reduced Ejection Fraction, with and Without Diabetes}

Sodium-glucose cotransporter 2 inhibitors (SGLT2i) are a class of oral medications currently approved for the treatment of diabetes mellitus. Empagliflozin, canagliflozin, and dapagliflozin comprise the currently available SGLT2i agents [26]. The EMPA-REG study, a randomized controlled trial of 7020 participants with diabetes and established CVD, showed a decrease in risk of the composite outcome of cardiovascular death, nonfatal myocardial infarction, and nonfatal stroke among individuals in the empagliflozin group compared with placebo (HR $0.86,95 \%$ CI $0.74-0.99, p=0.04$ for superiority) [27•]. Moreover, there was a significantly lower rate of cardiovascular death and heart failure hospitalization in the empagliflozin group compared with placebo. Findings from cardiovascular outcomes trials suggest that cardiovascular benefits, especially with respect to heart failure, are a class effect among SGLT2i [28, 29]. Since the original cardiovascular outcomes trials of SGLT2i, there has been immense interest in incorporating these agents in the treatment regimen for patients with heart failure with reduced ejection fraction (HFrEF). The rationale of the EMPEROR-Reduced study was to assess the efficacy of empagliflozin $10 \mathrm{mg}$ daily in the reduction of cardiovascular death or heart failure in patients with heart failure and with or without diabetes [5]. 


\section{Study Overview}

EMPEROR-Reduced was a randomized, placebo-controlled trial of 3730 patients, half of whom had diabetes mellitus. Participants were required to have class II, III, or IV heart failure and a left ventricular ejection fraction (LVEF) $\leq 40 \%$ on guideline-directed medical therapy. Participants with LVEF $\geq 30 \%$ also had to have a history of hospitalization for heart failure within the previous 12 months or a high level of N-terminal prohormone of brain natriuretic peptide (NTproBNP) $[\geq 1000 \mathrm{pg} / \mathrm{mL}$ in those with EF $31-35 \%, \geq$ $2500 \mathrm{pg} / \mathrm{mL}$ in those with EF of $36-40 \%$, or $\geq 600 \mathrm{pg} / \mathrm{mL}$ in those with $\mathrm{EF}$ of $\leq 30 \%]$. The primary outcome was a composite of cardiovascular death or heart failure hospitalization. Key secondary outcome measures included first and recurrent heart failure hospitalizations as well as decline in estimated glomerular filtration rate (eGFR).

Over a median follow-up of 16 months, individuals in the empagliflozin group had significantly lower primary outcome events compared with placebo (HR 0.75, 95\% CI 0.65-0.86, $p<0.001$; ARR 5.3\%). There was no heterogeneity of effect based on diabetes status. Total heart failure hospitalization events were significantly lower in the empagliflozin group compared with placebo: 10.7 versus 15.5 events/100 patientyr (HR $0.70,95 \%$ CI $0.58-0.85, p<0.001$ ), as was the annual rate of decline in eGFR $(-0.55 \mathrm{vs} .-2.28 \mathrm{~mL} / \mathrm{min} / 1.73 \mathrm{~m} 2$ of body-surface area/year, $p<0.001)$. There was no significant difference in death from cardiovascular cause or death from any cause between the empagliflozin and placebo groups. With respect to safety, uncomplicated genital tract infection was more frequent in the empagliflozin group compared with placebo ( $1.7 \%$ vs. $0.6 \%$ ), while rates of hypoglycemia and lower-limb amputation were not significantly different between groups.

\section{Clinical Implication}

The findings of EMPEROR-Reduced are consistent with the recent DAPA-HF trial, which assessed the efficacy of dapagliflozin in reduction of cardiovascular death or heart failure hospitalization in patients with HFrEF with or without diabetes mellitus [30•]. In EMPEROR-Reduced, the significance in the primary outcome was driven by a reduction in heart failure events. Importantly, there was a similar risk reduction noted among those with and those without diabetes mellitus. Current evidence suggests that empagliflozin confers significant cardiovascular and renal protection in patients with diabetes and HFrEF, showing reduction in risk for cardiovascular mortality and heart failure hospitalization. Results from EMPEROR-Reduced suggest that empagliflozin may also have a role in the treatment of HFrEF patients even without diabetes given the beneficial impact on heart failure hospitalization and renal outcomes.

\section{BRACE CORONA: Continuing Versus Suspending ACE Inhibitors and ARBs in COVID-19}

Underlying CVD or cardiovascular risk factors increase risk for morbidity and mortality in patients infected with COVID19 [31]. The renin-angiotensin-aldosterone system (RAAS) plays an important role in the mechanism of severe acute respiratory syndrome coronavirus 2 (SARS-CoV-2) tissue invasion and pathogenesis. SARS-CoV-2 is thought to enter cells via surface bound angiotensin-converting enzyme 2 (ACE2), which then leads to downregulation of surface ACE2 and accumulation of angiotensin II. Increased levels of angiotensin II are associated with vasoconstriction, fluid accumulation, and fibrosis [32].

There has been uncertainty regarding the use of angiotensin-converting enzyme inhibitors (ACEI) and angiotensin receptor blockers (ARB) in patients with COVID-19. On the one hand, ACEI/ARB may enhance viral binding and entry by increasing the expression of ACE2 receptors. On the other hand, diminishing the production of angiotensin II with ACEI/ARB and increased generation of angiotensin [7-13] may attenuate inflammation and fibrosis; thus, their use may be protective [33]. The rationale of the BRACE CORONA trial was to compare the impact on adverse outcomes between hospitalized COVID-19 patients who were continued on their chronic ACEI/ARB regimen versus those who had these medications temporarily held [6].

\section{Study Overview}

BRACE CORONA was a multi-center randomized trial of 659 patients hospitalized with a confirmed diagnosis of COVID-19 and who were on a chronic regimen of ACEI or ARB [34]. Patients who were hemodynamically unstable prior to confirmed diagnosis of COVID-19, those with decompensated heart failure hospitalization in the prior 12 months, those with more than 3 anti-hypertensive medications, and those using sacubitril-valsartan were excluded. Included participants were randomized 1:1 to either continued use of ACEI/ARB treatment or to temporarily suspending ACEI/ARB treatment for 30 days. The primary outcome was days alive and out of the hospital at 30 days.

At 30 days, $95.0 \%$ of the continuing ACEI/ARB group were alive and out of the hospital compared with $91.8 \%$ of the suspending ACEI/ARB group. There was no difference in primary outcome between the continuing ACEI/ARB (21.9 \pm 8.0 mean days) and suspending ACEI/ARB (22.9 7.1 mean days) groups (mean ratio $0.95,95 \%$ CI $0.90-1.01, p=0.09$ ). The mean difference between groups was $(-1.1$ days, $95 \% \mathrm{CI}$ $-2.33-0.17$ ). 


\section{Clinical Implication}

ACEI and ARB agents are well established, evidence-based therapy in the treatment of hypertension and heart failure. The BRACE CORONA trial provides clinical trial data showing that there is no clinical benefit in routinely withholding these agents in hospitalized patients with mild to moderate COVID19 infections. Therefore, unless otherwise indicated, ACEI/ARB therapy should be generally continued in patients with COVID-19.

\section{Conclusion}

The 2020 ESC Congress hosted several important clinical trials that demonstrate great strides within the field of CVD prevention and underscore the potential for further advancements.

\section{Compliance with Ethical Standards}

Conflict of Interest SSV: Grant Support: Department of Veterans Affairs, World Heart Federation, Tahir and Jooma Family. Honorarium: American College of Cardiology (Associate Editor for Innovations, acc. org).

SM: Personal fees from Amgen, Sanofi, Regeneron, Esperion, AstraZeneca, 89bio; Grants from Apple, Google, iHealth, Nokia, Maryland Innovation Initiative, American Heart Association, Aetna Foundation, PJ Schafer Memorial Fund, David and June Trone Family Foundation, Akcea Therapeutics, National Institutes of Health, outside the submitted work. In addition, SM has a pending patent on System of LDL-C Estimation. And he is a founder of and holds equity in Corrie Health, which intends to further develop the platform.

$\mathrm{XJ}$, MAR, AH, and AA do not have any relevant disclosures.

Human and Animal Rights and Informed Consent This article does not contain any studies with human or animal subjects performed by any of the authors.

\section{References}

Papers of particular interest, published recently, have been highlighted as:

- Of importance

•- Of major importance

1. Watts GF, editor RNAi inhibition of angiopoietin-like protein 3 (ANGPTL3) with ARO-ANG3 mimics the lipid and lipoprotein profile of familial combined hypolipidemia. ESC Congress 2020 Digital Exp; 2020.

2. Budoff MJ, Bhatt DL, Kinninger A, Lakshmanan S, Muhlestein JB, Le VT, et al. Effect of icosapent ethyl on progression of coronary atherosclerosis in patients with elevated triglycerides on statin therapy: final results of the EVAPORATE trial. Eur Heart J 2020.

3. Nidorf SM, Fiolet ATL, Mosterd A, Eikelboom JW, Schut A, Opstal TSJ, et al. Colchicine in Patients with Chronic Coronary Disease. N Engl J Med. 2020.
4. Rahimi K, editor BPLTTC - Blood pressure lowering for prevention of cardiovascular events across different levels of blood pressure. ESC Congress 2020 Digital Exp; 2020.

5. Packer M, Anker SD, Butler J, Filippatos G, Pocock SJ, Carson P, et al. Cardiovascular and renal outcomes with empagliflozin in heart failure. N Engl J Med. 2020.

6. Lopes RD, Macedo AVS, de Barros ESPGM, Moll-Bernardes RJ, Feldman A, D'Andrea Saba Arruda G, et al. Continuing versus suspending angiotensin-converting enzyme inhibitors and angiotensin receptor blockers: Impact on adverse outcomes in hospitalized patients with severe acute respiratory syndrome coronavirus 2 (SARS-CoV-2)-The BRACE CORONA Trial. Am Heart J. 2020;226:49-59.

7. Jia X, Al Rifai M, Gluckman TJ, Birnbaum Y, Virani SS. Highlights from selected cardiovascular disease prevention studies presented at the 2019 European Society of Cardiology Congress. Curr Atheroscler Rep. 2019;21(12):46.

8. Al Rifai M, Jia X, Al-Mallah MH, Miedema MD, Martin SS, Virani SS. Major randomized clinical trials in cardiovascular disease prevention presented at the 2019 American College of Cardiology Annual Scientific Session. Curr Atheroscler Rep. 2019;21(8):31.

9. Jia X, Al Rifai M, Liu J, Agarwala A, Gulati M, Virani SS. Highlights of studies in cardiovascular disease prevention presented at the 2020 American College of Cardiology Annual Scientific Session. Curr Atheroscler Rep. 2020;22(8):32.

10. Stitziel NO, Khera AV, Wang X, Bierhals AJ, Vourakis AC, Sperry $\mathrm{AE}$, et al. ANGPTL3 deficiency and protection against coronary artery disease. J Am Coll Cardiol. 2017;69(16):2054-63.

11. Jorgensen AB, Frikke-Schmidt R, Nordestgaard BG, TybjaergHansen A. Loss-of-function mutations in APOC 3 and risk of ischemic vascular disease. N Engl J Med. 2014;371(1):32-41.

12. Hussain A, Ballantyne CM, Saeed A, Virani SS. Triglycerides and ASCVD risk reduction: recent insights and future directions. Curr Atheroscler Rep. 2020;22(7):25.

13. Nordestgaard BG, Nicholls SJ, Langsted A, Ray KK, TybjaergHansen A. Advances in lipid-lowering therapy through genesilencing technologies. Nat Rev Cardiol. 2018;15(5):261-72.

14. Ballantyne CM, editor RNA interference targeting apolipoprotein C-3 with ARO-APOC3 in healthy volunteers mimics lipid and lipoprotein findings seen in subjects with inherited apolipoprotein C-3 deficiency. ESC Congress 2020 Digital Exp; 2020.

15.• Cholesterol Treatment Trialists C, Baigent C, Blackwell L, Emberson J, Holland LE, Reith C, et al. Efficacy and safety of more intensive lowering of LDL cholesterol: a meta-analysis of data from 170,000 participants in 26 randomised trials. Lancet. 2010;376(9753):1670-81 Meta-analysis of major statin trials evaluating the efficacy and safety of LDL-C lowering using statins.

16. Brautbar A, Leary E, Rasmussen K, Wilson DP, Steiner RD, Virani S. Genetics of familial hypercholesterolemia. Curr Atheroscler Rep. 2015;17(4):491.

17. Bhatt DL, Steg PG, Miller M, Brinton EA, Jacobson TA, Ketchum $\mathrm{SB}$, et al. Cardiovascular risk reduction with icosapent ethyl for hypertriglyceridemia. N Engl J Med. 2019;380(1):11-22 Large randomized controlled trial assessing the efficacy and safety of icosapent ethyl in risk reduction of ASCVD.

18. Zeb I, Li D, Nasir K, Malpeso J, Batool A, Flores F, et al. Effect of statin treatment on coronary plaque progression - a serial coronary CT angiography study. Atherosclerosis. 2013;231(2):198-204.

19. Nicholls SJ, Puri R, Anderson T, Ballantyne CM, Cho L, Kastelein $\mathrm{JJ}$, et al. Effect of evolocumab on progression of coronary disease in statin-treated patients: the GLAGOV randomized clinical trial. JAMA. 2016;316(22):2373-84.

20.• Grundy SM, Stone NJ, Bailey AL, Beam C, Birtcher KK, Blumenthal RS, et al. 2018 AHA/ACC/AACVPR/AAPA/ABC/ $\mathrm{ACPM} / \mathrm{ADA} / \mathrm{AGS} / \mathrm{APhA} / \mathrm{ASPC} / \mathrm{NLA} / \mathrm{PCNA}$ guideline on the 
management of blood cholesterol: a report of the American College of Cardiology/American Heart Association task force on clinical practice guidelines. Circulation. 2019;139(25):e1082-e143 Contemporary guidelines on the management of blood cholesterol in primary and secondary prevention of ASCVD.

21. Virani SS, Polsani VR, Nambi V. Novel markers of inflammation in atherosclerosis. Curr Atheroscler Rep. 2008;10(2):164-70.

22. Ridker PM, Everett BM, Thuren T, MacFadyen JG, Chang WH, Ballantyne C, et al. Antiinflammatory therapy with Canakinumab for atherosclerotic disease. N Engl J Med. 2017;377(12):1119-31 Large randomized contolled trial demonstrating that the targeting of inflammation with canakinumab in patients at high risk for ASCVD and with increased inflammation was associated with ASCVD risk reduction.

23. Nidorf SM, Eikelboom JW, Budgeon CA, Thompson PL. Lowdose colchicine for secondary prevention of cardiovascular disease. J Am Coll Cardiol. 2013;61(4):404-10.

24. Tardif JC, Kouz S, Waters DD, Bertrand OF, Diaz R, Maggioni AP, et al. Efficacy and safety of low-dose colchicine after myocardial infarction. N Engl J Med. 2019;381(26):2497-505 Large randomized controlled trial assessing efficacy and safety of low dose colchicine in ASCVD risk reduction after acute myocardial infarction.

25.• Whelton PK, Carey RM, Aronow WS, Casey DE Jr, Collins KJ, Dennison Himmelfarb C, et al. 2017 ACC/AHA/AAPA/ABC/ ACPM/AGS/APhA/ASH/ASPC/NMA/PCNA guideline for the prevention, detection, evaluation, and Management of High Blood Pressure in adults: a report of the American College of Cardiology/American Heart Association task force on clinical practice guidelines. Hypertension. 2018;71(6):e13-e115 Contemporary guidelines on the management of blood pressure in adults.

26. Jia X, Mehta PB, Ye Y, Alam M, Birnbaum Y, Bajaj M. SGLT2 inhibitors and cardiovascular outcomes: current perspectives and future potentials. Curr Diab Rep. 2018;18(9):63.

27. Zinman B, Wanner C, Lachin JM, Fitchett D, Bluhmki E, Hantel S, et al. Empagliflozin, cardiovascular outcomes, and mortality in type
2 diabetes. N Engl J Med. 2015;373(22):2117-28 Large cardiovascular outcomes trial of empagliflozin.

28. Neal B, Perkovic V, Mahaffey KW, de Zeeuw D, Fulcher G, Erondu N, et al. Canagliflozin and cardiovascular and renal events in type 2 diabetes. N Engl J Med. 2017;377(7):644-57 Large cardiovascular outcomes trial of canagliflozin.

29. Wiviott SD, Raz I, Bonaca MP, Mosenzon O, Kato ET, Cahn A, et al. Dapagliflozin and cardiovascular outcomes in type 2 diabetes. N Engl J Med. 2019;380(4):347-57 Large cardiovascular outcomes trial of dapagliflozin.

30. McMurray JJV, Solomon SD, Inzucchi SE, Kober L, Kosiborod MN, Martinez FA, et al. Dapagliflozin in patients with heart failure and reduced ejection fraction. N Engl J Med. 2019;381(21):19952008 Randomized controlled trial assessing efficacy and safety of dapagliflozin in patients with heart failure with reduced ejection fraction and with or without diabetes mellitus.

31. Clerkin KJ, Fried JA, Raikhelkar J, Sayer G, Griffin JM, Masoumi A, et al. COVID-19 and cardiovascular disease. Circulation. 2020;141(20):1648-55.

32. Vaduganathan M, Vardeny O, Michel T, McMurray JJV, Pfeffer MA, Solomon SD. Renin-angiotensin-aldosterone system inhibitors in patients with Covid-19. N Engl J Med. 2020;382(17): 1653-9.

33. Mancia G, Rea F, Ludergnani M, Apolone G, Corrao G. Reninangiotensin-aldosterone system blockers and the risk of Covid-19. N Engl J Med. 2020;382(25):2431-40.

34. Lopes RD, editor Continuing versus suspending angiotensinconverting enzyme inhibitors and angiotensin receptor blockers: impact on adverse outcomes in hospitalized patients with severe acute respiratory distress syndrome coronavirus 2 (SARS-CoV-2). ESC Congress 2020 Digital Exp; 2020.

Publisher's Note Springer Nature remains neutral with regard to jurisdictional claims in published maps and institutional affiliations. 